\title{
Granite Strain Waveform Curve Analysis in SHPB Experiment
}

\author{
Qingcheng ZHANG ${ }^{1,2, a}$, Dianshu $\mathrm{LIU}^{2, \mathrm{~b}}$, Shenglin $\mathrm{LI}^{2, \mathrm{c}}$ and \\ Shufeng LIANG ${ }^{2, d}$ \\ ${ }^{1}$ China university of mining \&technology (Beijing) school of mechanical and architectural \\ engineering, CHINA \\ ${ }^{2} \mathrm{JCHX}$ Mining management Co.,LTD., CHINA \\ afamilyzqcking@126.com, ${ }^{\mathrm{b}} 376360616 @ q q . c o m,{ }^{\mathrm{c}}$ shenglinli@sina.com, \\ d122577671@qq.com
}

Keywords: granite; SHPB; bullet length; strain wave; damage

Abstract. The damage degree of the rock and its strain has the nature relation, this paper choose the granite which is most common rock in the engineering practice as the research object. It carried on more categories of impact test research about granite specimen of length to diameter ratio is 0.8 times in using split hopkinson pressure bar (SHPB) device, and inferred the measurement of rock damage degree is mainly decided by the reflected wave waveform. Along with the rising of the impact energy of bullets, the measured waveform of reflected wave from development to the "W", "V" type and "U" type on compression bar. When the rock is damage, the reflection waveform more appears as "U" and "W" type. It can be concluded that the development and distribution of the internal cracks of rock determines the diversity of the reflection wave shape, and many types of the reflected wave shape also reflected the loading condition and the relationship between the characteristics of rock.

\section{Introduction}

Since the B.Hopkinson SHPB device prototype was proposed, the pressure bar is divided into two segments by Kolsky ${ }^{[1]}$, putting the specimen in the middle of their input and output bar, it can be measured the material stress-strain relationship under impact load by this device, so the elastic compression bar in SHPB device also can be called the Kolsky bar. In the early 1970s, triaxial SHPB experimental device has been designed successively by Lindholm ${ }^{[2]}$ and Christensen ${ }^{[3]}$, in the confining pressure of rock under dynamic test which is concluded that with the increase of confining pressure, fracture strength increases linearly. With the increase of strain rate, the failure strength also rises at the same time in certain conditions of confining pressure. Yalun $\mathrm{YU}^{[4]}$ used the three axis SHPB experimental apparatus, the characteristics of the rock under dynamic loading, mechanism concept of micro-damage and damage mechanism was studied and the establishment of appropriate constitutive equation. Now split hopkinson (SHPB) test technology has become a standard method has been widely applied in the strain rate is $10 \mathrm{~s}^{-1} \sim 104 \mathrm{~s}^{-1}$ material within the scope of the dynamic mechanical properties test ${ }^{[5]}$. Hult and Broberg ${ }^{[6]}$ in Sweden, the British of Hayhurst ${ }^{[7]}$ and Leckie ${ }^{[8]}$, the American Krajcinovic ${ }^{[9]}$ created a geometry damage theory, mainly relays on the phenomenological research methods, it considers material damage is mainly caused by material inside the various micro defects, so the damage size and the damage evolution law of the material shape are effected by the size and density of micro defects, but also be to the way of distribution, which is the damage of the rock is the result of the internal structure of the defects of 
the rocks. But it did not show that these defects under the action of external force in what form the rock damage. This article is based on SHPB pressure bar device, choosing the granite specimen of length to diameter ratio is 0.8 times, finding the suitable characterization parameter or expression for rock damage under the action of one dimensional stress in more categories of impact load test.

\section{Test Device}

Damage evolution law of rock under the action of stress wave and rock damage state is a hot spot of engineering and academic research in current, in order to study the dynamic mechanical properties of the rock, using split hopkinson pressure bar (SHPB) experiments for dynamic mechanical impact test. SHPB device consists of power system, the bullet, input shaft and output shaft, absorption and test records system, the specimen in the study on input shaft and output shaft. There is the laboratory of split hopkinson pressure bar (SHPB) system device in China university of mining \& technology (Beijing) hopkinson pressure bar. In concluding phi $75 \mathrm{~mm}, 50 \mathrm{~mm}$ and 14.5 $\mathrm{mm}$ three series of compression bar, the bullet length is $400 \mathrm{~mm}, 600 \mathrm{~mm}, 800 \mathrm{~mm}$ and $1000 \mathrm{~mm}$, two kinds materials of the bar which are the aluminum and steel, it can be satisfied the dynamic testing of different test materials. Huang Chaojun ${ }^{[10]}$ systematically studied the effect of the length of the limestone specimen, and concluded that the length to diameter ratio of 0.8 can well reflect the dynamic mechanical properties of rock. This paper tests the granite specimen length to diameter ratio are selected as 0.8 , the length of the specimen is $40 \mathrm{~mm}, 50 \mathrm{~mm}$ diameter, which can guarantee in the experiment to ignore transverse wave effect of the specimens, and can have enough balance time to collect data on the waveform.

\section{Shock Waveform Curve Analysis}

In order to ensure the granite specimen is not broken through three times impact in this series tests, the velocity of bullet must be relatively low. The kinds length of bullet are $400 \mathrm{~mm}, 600 \mathrm{~mm}, 800$ $\mathrm{mm}$ and $1000 \mathrm{~mm}$, the velocity of bullet are divided into five gradient sections which are decided by SHPB device control accuracy and test requirements. Detailed comparative analysis the relationship which is between different stress wave effect of rock damage and the strain wave. The strain wave of $400 \mathrm{~mm}$ length bullets is showed in figure 1 .

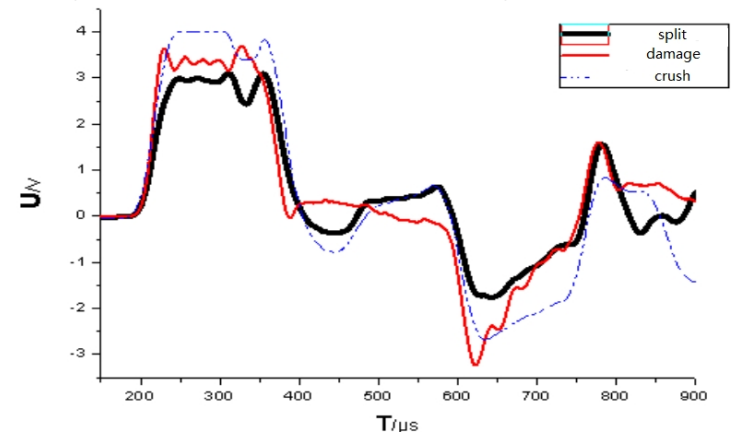

( a ) The incident wave and reflected wave

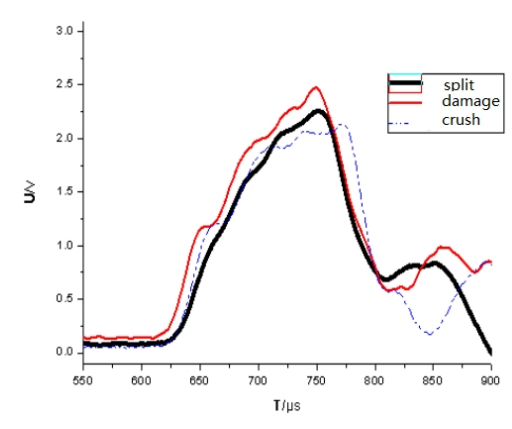

( b ) Transmission wave

Fig 1 The incident and transmission wave of bullet length of $400 \mathrm{~mm}$

Figure 1(a) and 1(b) are showed that the incident wave shapes which are the approximation of the rectangular is stable. It is satisfied the one dimensional elastic stress wave theory, namely the bullet length decides stress wave duration, it will not change with the changes in the velocity of the bullet. Incident wave amplitude value is determined by the bullet impact velocity, by a large number of 
research data shows a linear relationship. In the range of allowable error, measured by the incident wave amplitude value is consistent with the theory of stress wave. Transmission amplitude value also increases as the incident wave amplitude. Due to the energy of rock to absorb some of the stress wave and a portion of the energy dissipation in the process of stress wave, the transmission wave amplitude is always smaller than the incident wave amplitude.

These reflected waves have certain consistency in rise period, the reflection wave behind most of the wave shape is associated with the bullet impact velocity and rock damage degree. When the rock specimen is little damage by the stress wave, the transmission wave rapidly come into the platform, and then quickly reduced to 0 , at the same time, the transmitted waveform and the incident wave have good consistency; When the damage of rock is bigger, the transmission wave rises slowly, the platform period is short, the falling gradient is fast. Reflection wave and the horizontal axis which are enclosed area is smaller, the transmission wave and the horizontal axis that are enclosed area is larger, the damage degree of the rock is lower. When judging the damage degree of the rock can be transmitted wave and reflected wave is presupposed.

Most of rock specimens reflected wave into a "V" shape that don't see naked eye visible on the surface of the rock crack. When there is tiny broken crack face, a handful of rock specimen of reflected wave force is "W" shape, but the second trough is very shallow. Many rock specimen of reflected wave are opening a large " $\mathrm{V}$ " shape, the causes of this situation is the rock face after stress wave action and internal crack distribution form and the number of the crack is different, every time the stress wave in the rock face of reflection and transmission are not the same, and it is possible that the stress wave in this position have also been dried radiation and diffraction. It can also be understood as the incident wave generated in the bullet for rectangular pulse stress wave, it is composed of multiple variety frequency sine wave superposition, the frequency of the wave in the rock face and part of internal cracks is out, due to the diversity of the reflection wave during the second half of the waveform.
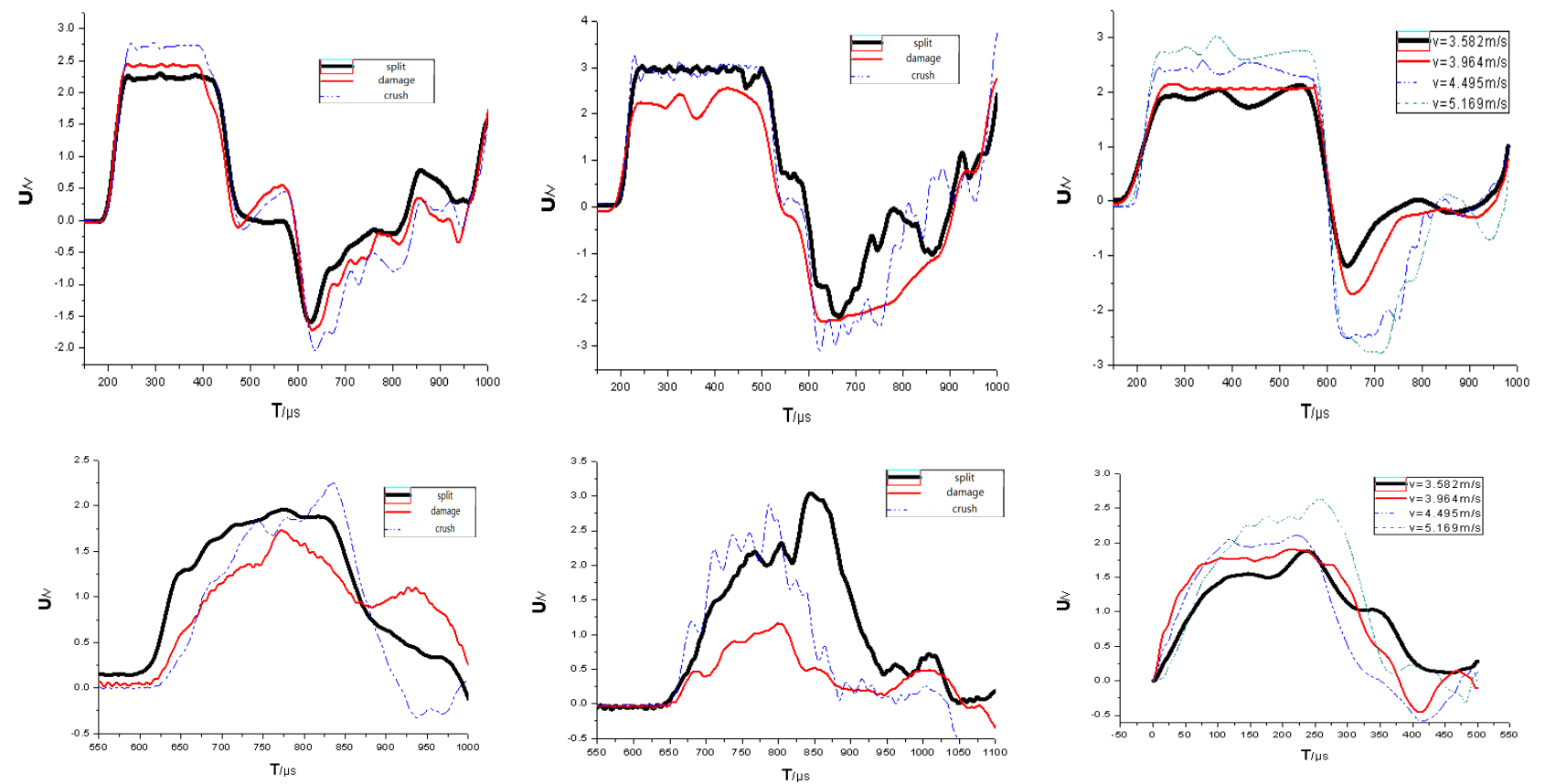

Fig 2 The incident and transmission wave of bullet length of 600,800 and $1000 \mathrm{~mm}$

When the reflection waveform is " $U$ " type, the rock specimen is almost broken.. In fact, the reflection wave is more like the shape of the incident wave in this time, the reflected wave trough is 
a slash and not flat, because the rocks have been damaged need to consume some impact energy. It also can be caused by more reflection of the diversity of the shape of the transmitted wave at the same time. Figure 2 are showed that the bullet is $600 \mathrm{~mm}, 800 \mathrm{~mm}$ and $1000 \mathrm{~mm}$ of strain wave and the bullet for $400 \mathrm{~mm}$ are the similar between strain wave and the granite damaged the relations. In the case of a similar incident wave amplitude, the longer the length of bullet, the more the stress wave effect. When the waveform and the time ax into the shape of the area is larger, the incident energy crease, the greater the reflection and transmission can also increase, specimen damage degree is more serious. The stress wave wavelength also plays an important role in the process of granite damage, but only when the damage reaches a certain degree of the rock, the wavelength begins to work on rock breakage. The rock damage is a result of internal structure fracture, it can be characterized by reflection waveform.

\section{Conclusions}

(1) When the bullet length do not change, the time of stress wave is the constant value. It will not change with the changes of the velocity of the bullet. And it combined with stress wave theory, also indirectly proves that the accuracy of the test data.

(2) The granite damage degree is mainly decided by the reflected wave waveform. Along with the rising of the impact energy of bullets, the reflected wave is from a "V" type to "W", for "U". Namely the damage degree of the rock can be characterized by reflection waveform.

(3) When the rock is broken, the reflection type shows the shape of " $U$ " and "W" mostly, it is caused by the development and distribution of the internal cracks of rock determines the diversity of the reflection wave shape. The shape also reflects relationship between the loading condition and the characteristics of rock.

\section{References}

[1] KolskyH. An Investigation of the Mechanical Properties of Materials at Very High Rates of Loading[J]. Proceedings of the Physical Society of London , 1949 , B62 : 676-700.

[2] Christensen R J, Swanson S R.Brown W S.Split Hopkinson Bar tests on rocks under confining pressure[J].Experimental Mechnics , 1973 , 12(12) : 508-513.

[3] Lindholm U S.Yearkly L M , Nagy A.The dynamic strength and fracture properties of dresser basalt[J].IntJ Rock MechSci and GeomechAbstr , 1974 , (11) : 181-191.

[4] Yu Yalun. Triaxial SHPB device is used to study the dynamic load characteristics of the rock [J]. Journal of geotechnical engineering, 1992, 14 (3) : 76-79.

[5] FieldJE, WalleySM, ProudWG. etal. Review of experimental techniques for high rate deformation and shock studies[J]. International Journal of Impact Engineering, 2004, 30 ( 7):725-775

[6] Hult,J. Damage induced tensile instability. 1975. Proceeding of ICM-3. London.

[7] Hayhurst, D.R.. Estimates of the creep rupture life time of structures using the finite element 
method. 1975. Journal of Mechanics and Physics of Solids. 23:132 143

[8] Leckie , F.A.. Tensorial nature of damage measuring internal variables. 1980. Proceedings of IUTAM Symposium on Physical Non-Linearties in Structural Analysis. Springer-Verlag.

[9] Krajcinovic, D. and Lemaitre,J. Continuum damage mechanics theory and applications. 1987. U.S.A.: Spring-Verlag

[10] Huang Chaojun, The damage and evolution of the stress wave under the action of lime experimental study on rock [D], China university of mining \&technology (Beijing), 2009 